\title{
Heart Failure and Cardiac Events: Is a Consecutive Measurement of Biomarkers a Simple and Practical Approach?
}

\author{
José Miguel Rivera-Caravaca ${ }^{1,2}$ María Asunción Esteve-Pastor ${ }^{1[(])}$
}

${ }^{1}$ Department of Cardiology, Hospital Clínico Universitario Virgen de la Arrixaca, Instituto Murciano de Investigación Biosanitaria (IMIB-Arrixaca), Centro de Investigación Biomédica en Red-Enfermedades Cardiovasculares (CIBERCV), University of Murcia, Murcia, Spain

${ }^{2}$ Faculty of Nursing, Catholic University of Murcia (UCAM), Murcia, Spain

Thromb Haemost 2019;119:1891-1893.

Heart failure (HF) is common, with a prevalence of approximately $2 \%$ in the overall population, and increasing to 5 to $9 \%$ in people older than 65 years. ${ }^{1}$ This prevalence is likely to increase in relation to an aging population, increasing prevalence of comorbidities or risk factors, and improved survival of postmyocardial infarction. ${ }^{2}$ Of note, $\mathrm{HF}$ is associated with worse clinical outcomes, including successive hospitalizations, higher risk of mortality, and impaired quality of life. ${ }^{3,4}$

Over the last decade, the role of various biomarkers for understanding underlying pathophysiology, improving risk stratification, or prediction of adverse events in cardiovascular diseases has gained much attention. ${ }^{5-11}$ In the clinical setting, specifically in HF, the natriuretic peptides (NPs) (B-type natriuretic peptide [BNP], N-terminal fraction of BNP [NT-proBNP], and midregional proANP) are markers of myocardial stress that have been widely investigated, being predictive of mortality and rehospitalization. ${ }^{12-14}$ Similarly, cardiac troponins are markers of myocardial injury that have been observed to be elevated in many HF patients even in the absence of an acute coronary syndrome due to the stress and damage of myofibrillar proteins. Hence, NP and troponins concentrations may help in the diagnosis and prognosis of $\mathrm{HF}^{15-17}$

The prothrombotic state of HF has been well described, whereby the presence of hemostasis, platelets, and endothelial function abnormalities confers an increased risk of thrombogenesis. Hence, some markers associated with thrombogenesis and endothelial dysfunction may be useful in identifying "high-risk" HF patients and have prognostic implications. ${ }^{14,18}$

In this issue of Thrombosis and Haemostasis, van den Berg et al investigate some fibrinolytic factors scarcely explored in $\mathrm{HF}$, plasminogen activator inhibitor 1 (PAI-1), tissue-type plasminogen activator (tPA), urokinase-type PA (uPA), and

received

October 8, 2019

accepted after revision

October 8, 2019

Address for correspondence José Miguel Rivera-Caravaca, RN, PhD, Department of Cardiology, Hospital Clínico Universitario Virgen de la Arrixaca, Ctra. Madrid-Cartagena s/n, Murcia, 30120, Spain (e-mail: jmrivera429@gmail.com).

soluble urokinase PA surface receptor (suPAR). In their study, while tPA concentrations were not related with the endpoint of interest, longitudinally measured PAI- 1 , uPA, and suPAR were strongly associated with adverse cardiac events in patients with chronic HF from the Bio-SHiFT cohort. ${ }^{19}$

Some years ago, a report from the Ludwigshafen Risk and Cardiovascular Health study demonstrated that the tPA/PAI-1 complex concentration had additional prognostic value above and beyond NT-proBNP and was an independent predictor of mortality from all-cause and cardiovascular in patients with $\mathrm{HF}$ with preserved ejection fraction. ${ }^{14}$ Although these results were derived from a long period of observation (more than 9 years), they are based on baseline measurement of tPA/PAI-1 complex. In fact, one common criticism of biomarker studies is that they measure biomarkers only once, often at baseline, and in highly selected trial cohorts. Nevertheless, as van den Berg et al correctly state in their manuscript, plasma levels of coagulation and fibrinolysis factors vary over time. Even during the same day biomarkers concentrations could be different, due to circadian variability, the hemodynamic state of the patient, and concomitant diseases, which highlights the role of the differential gradient of two measurements.

Thus, how can a baseline measurement of a biomarker predict adverse events many years later? Such an approach is controversial, even when this measurement shows "statistical differences." Indeed, the risk of adverse events is not static, but dynamic. During a follow-up period, this risk is usually modified by aging, incident or changing comorbidities, and changes in drug therapies. Accordingly, biomarkers are also modified, and therefore, in proximity to the adverse event, biomarker levels might be quite "different" from measured baseline levels (-Fig. 1).

(c) 2019 Georg Thieme Verlag KG Stuttgart · New York
DOI https://doi.org/ 10.1055/s-0039-3400274. ISSN 0340-6245. 


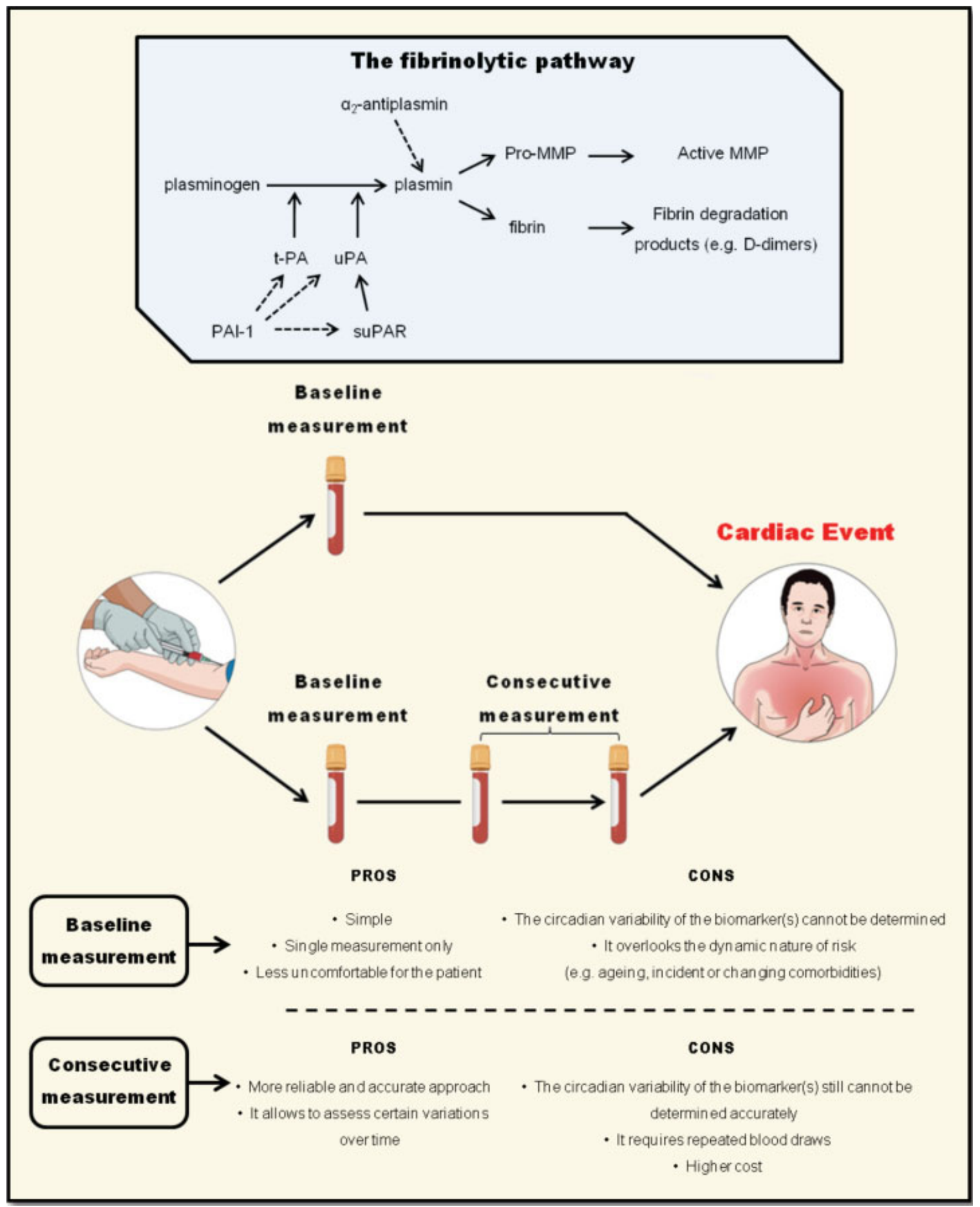

Fig. 1 The fibrinolytic pathway and different approaches for assessing biomarkers. MMP, matrix metalloproteinase; PAI-1, plasminogen activator inhibitor 1; suPAR, soluble urokinase plasminogen activator surface receptor; tPA, tissue-type plasminogen activator; uPA, urokinasetype plasminogen activator.

For the above reasons, the study by van den Berg et al has important strengths. The study was performed in a prospective and observational real-world cohort, which better reflects real-world clinical practice. ${ }^{20}$ Of note, they measured biomarkers every 3 months over more than 2 years, and by using this approach, the authors were able to select the last two measurements prior to the endpoint, that is, closer in time to the adverse event. This provides more reliable and accurate information about the status of biomarkers, and their potential association with the event(s) of interest. Specifically, they demonstrated how important it is to take into account the change in biomarker levels with laboratory follow-up samples, particularly when the aim is to aid in decision making or risk stratification.

Nevertheless, several issues need to be clarified and further investigated. For example, many biomarkers are nonspecific and therefore can be associated with various cardiovascular and noncardiovascular outcomes at the same 
time, including thromboembolism, bleeding, decompensated $\mathrm{HF}$, atrial fibrillation, myocardial infarction, renal failure, severe infection, inflammatory disorders, or death. Thus, whether biomarkers are actually related to worse outcomes per se or simply reflect sick patients or sick hearts is still unknown. The inter- and intrapatient variability, use of specific biomarker assays, diurnal variation, influence of concomitant diseases, and drug therapies, as well as access to laboratories in different health care systems, may also hinder the generalizability of using some biomarkers. ${ }^{21}$

In conclusion, it is not just about "statistical significance" but the necessity of practical usefulness. Measurement of biomarkers should also balance costs and daily use in clinical practice since, and importantly, the incremental predictive value of biomarkers over simple clinical factors is only marginal even though multiple biomarkers are added. ${ }^{22}$ In busy clinics and emergencies or ward settings, simplicity and practicality matter.

\section{Conflict of Interest}

None declared.

\section{References}

1 Benjamin EJ, Blaha MJ, Chiuve SE, et al; American Heart Association Statistics Committee and Stroke Statistics Subcommittee. Heart Disease and Stroke Statistics-2017 update: a report from the American Heart Association. Circulation 2017;135(10):e146-e603

2 GBD 2013 Mortality and Causes of Death Collaborators. Global, regional, and national age-sex specific all-cause and cause-specific mortality for 240 causes of death, 1990-2013: a systematic analysis for the Global Burden of Disease Study 2013. Lancet 2015;385(9963):117-171

3 Göbel S, Prochaska JH, Eggebrecht L, et al. Management of oral anti-coagulation in patients with heart failure-insights from the ThrombEVAL study. Thromb Haemost 2018;118(11):1930-1939

4 Shah KS, Xu H, Matsouaka RA, et al. Heart failure with preserved, borderline, and reduced ejection fraction: 5-year outcomes. J Am Coll Cardiol 2017;70(20):2476-2486

5 Esteve-Pastor MA, Roldán V, Rivera-Caravaca JM, Ramírez-Macías I, Lip GYH, Marín F. The use of biomarkers in clinical management guidelines: a critical appraisal. Thromb Haemost 2019;119(12): 1901-1919

6 Sarhene M, Wang Y, Wei J, et al. Biomarkers in heart failure: the past, current and future. Heart Fail Rev 2019;24(06):867-903

7 Sulzgruber P, Koller L, Winter MP, et al. The impact of $\mathrm{CD} 4{ }^{+} \mathrm{CD} 28^{\text {null }}$ T-lymphocytes on atrial fibrillation and mortality in patients with chronic heart failure. Thromb Haemost 2017;117 (02):349-356

8 Richter B, Koller L, Hohensinner PJ, et al. Fractalkine is an independent predictor of mortality in patients with advanced heart failure. Thromb Haemost 2012;108(06):1220-1227

9 Zakai NA, Olson NC, Judd SE, et al. Haemostasis biomarkers and risk of intracerebral haemorrhage in the REasons for Geographic and Racial Differences in Stroke Study. Thromb Haemost 2017; 117(09):1808-1815

10 López P, Rodríguez-Carrio J, Martínez-Zapico A, et al. Serum levels of anti-PON1 and anti-HDL antibodies as potential biomarkers of premature atherosclerosis in systemic lupus erythematosus. Thromb Haemost 2017;117(11):2194-2206

11 Mjelva OR, Svingen GFT, Pedersen EKR, et al. Fibrinogen and neopterin is associated with future myocardial infarction and total mortality in patients with stable coronary artery disease. Thromb Haemost 2018;118(04):778-790

12 Molvin J, Jujic A, Bachus E, et al. Cardiovascular biomarkers predict post-discharge re-hospitalization risk and mortality among Swedish heart failure patients. ESC Heart Fail 2019. Doi: 10.1002/ehf2.12486

13 Stienen S, Salah K, Moons AH, et al. NT-proBNP (N-terminal pro-Btype natriuretic peptide)-guided therapy in acute decompensated heart failure: PRIMA II randomized controlled trial (can NT-ProBNPguided therapy during hospital admission for acute decompensated heart failure reduce mortality and readmissions?) Circulation 2018; 137(16):1671-1683

14 Winter MP, Kleber ME, Koller L, et al. Prognostic significance of tPA/PAI-1 complex in patients with heart failure and preserved ejection fraction. Thromb Haemost 2017;117(03):471-478

15 Mueller C, McDonald K, de Boer RA, et al; Heart Failure Association of the European Society of Cardiology. Heart Failure Association of the European Society of Cardiology practical guidance on the use of natriuretic peptide concentrations. Eur J Heart Fail 2019;21(06): 715-731

16 Ponikowski P, Voors AA, Anker SD, et al; ESC Scientific Document Group. 2016 ESC guidelines for the diagnosis and treatment of acute and chronic heart failure: the Task Force for the diagnosis and treatment of acute and chronic heart failure of the European Society of Cardiology (ESC)Developed with the special contribution of the Heart Failure Association (HFA) of the ESC. Eur Heart J 2016;37(27):2129-2200

17 Yancy CW, Jessup M, Bozkurt B, et al. 2017 ACC/AHA/HFSA focused update of the 2013 ACCF/AHA guideline for the management of heart failure: a report of the American College of Cardiology/American Heart Association Task Force on Clinical Practice Guidelines and the Heart Failure Society of America. J Am Coll Cardiol 2017;70(06):776-803

18 Lip GY, Gibbs CR. Does heart failure confer a hypercoagulable state? Virchow's triad revisited. J Am Coll Cardiol 1999;33(05): 1424-1426

19 van den Berg VJ, Bouwens E, Umans VAWM, et al. Longitudinally measured fibrinolysis factors are strong predictors of clinical outcome in patients with chronic heart failure - the Bio-SHiFT study. Thromb Haemost 2019;119(12):1947-1955

20 Rivera-Caravaca JM, Esteve-Pastor MA, Marín F, et al. A propensity score matched comparison of clinical outcomes in atrial fibrillation patients taking vitamin $\mathrm{K}$ antagonists: comparing the "real-world" vs clinical trials. Mayo Clin Proc 2018;93(08): 1065-1073

21 Kozieł M, Potpara TS, Lip GYH. Using blood biomarkers to identify atrial fibrillation-related stroke. Stroke 2019;50(08):1956-1957

22 Rivera-Caravaca JM, Marín F, Vilchez JA, et al. Refining stroke and bleeding prediction in atrial fibrillation by adding consecutive biomarkers to clinical risk scores. Stroke 2019;50(06):1372-1379 